\title{
Discussion on Interior Design Based on Ecological Theory
}

\section{Yunpeng Li}

Academy of Fine Arts, Qinghai normal University, Xining, Qinghai province, China 810008.

\begin{abstract}
At present, the problem of material waste and environmental damage in interior decoration design is very obvious. Obviously, this runs counter to the current sustainable development concept. The emergence of green interior design improves this problem. From the perspective of ecological theory and health and environmental protection From the above point of view, green interior design gives consumers better ecological enjoyment and natural experience. This article discusses interior design from the perspective of ecological theory.
\end{abstract}

Keywords: Ecological Theory; Interior Design; Sustainable Development

With the continuous deepening of the concept of sustainable development, more and more consumers attach great importance to the environmental protection of materials when designing interiors. With the continuous improvement of social and economic levels, consumers' aesthetic awareness continues to increase. They began to pay attention to whether the interior design can reflect the characteristics of humanization, so the green and environmentally friendly interior design has been widely recognized. This is a very new concept for the new era of housing design. New technologies can not only contribute to people's lives. Bring convenience, while truly making consumers feel sustainable development.

\section{Ecological interior design principles}

\subsection{Living health principles}

The interior is the main focus of the human environment, and it is also the environment most exposed to human beings. Therefore, the most important and core point of the requirements for interior design is living health. The socalled health includes two aspects, namely physical health and mental health. The principle that designers should follow when designing interiors is mainly to protect human health. Surveys have shown that if there are pollution sources indoors, it will inevitably have a negative effect on people's health. Therefore, the interior design under the ecological concept requires that all indoor pollution sources should be eliminated, and the materials must be guaranteed to have no impact on human health. In order to be consistent with human mental health, the lighting, air circulation, temperature and humidity in the interior design must also be Meet the needs of residents, so as to build a comfortable environment belonging to the residents, making them feel relaxed and happy.

\subsection{Aesthetic principles}

Regarding interior design, in addition to requiring health and harmlessness, occupants also have higher requirements for indoor aesthetics, especially the penetration of ecological culture in various fields. The so-called ecological design does not only represent the natural pollution-free indoor environment, but can be combined with living According to the needs of consumers, make reasonable plans for the use of the house, and use different styles to show the aesthetics of interior style design. At present, the style of interior design is not only limited to industrial style, but more to show natural style and minimalism. Ism.

The aesthetic principles of indoor ecological design are also reflected in the harmonious beauty between humans and

Copyright (C) 2020 Yunpeng Li

doi: $10.18282 /$ le.v9i7.1515

This is an open-access article distributed under the terms of the Creative Commons Attribution Non-Commercial License

(http://creativecommons.org/licenses/by-nc/4.0/), which permits unrestricted non-commercial use, distribution, and reproduction in any medium, provided the original work is properly cited. 
nature. In order to reflect the natural beauty, it is necessary to focus on the use of new technologies and new materials and energy. Of course, the occupants with the conditions also pursue higher levels of beauty. Aesthetics often need some light and shadow changes and temperature changes to cooperate, which means that the current interior design wants to pursue a higher level of enjoyment in ecological aesthetics, and does not need to give up other beauty due to natural harmony.

\subsection{Ecological characteristics}

Compared with ordinary interior design, the biggest feature of ecological interior design is that it can reflect the ecology in the design. The ecology mentioned here is actually sustainable development. Ecological interior design can effectively protect human health and aesthetics. , The Chinese style and resources I selected are all renewable resources. The design is simple and reasonable, and the selection of materials is reasonable. Ensuring resource conservation is the most important method in ecological interior design. The interior designer will make reasonable space Arrangement, eliminate all extravagant design styles, combine the needs of users, and restructure the interior under the principles of space design.

When choosing interior decoration, in order to ensure its ecological and environmental protection and meet the requirements of aesthetic design, designers should avoid choosing cherished non-renewable materials and replace them with sustainable materials. In addition, when choosing energy sources, solar wind energy should be selected first, and the indoor lighting design should also make more scientific use of natural lighting to ensure the environmental protection of the interior design.

\section{Ecological interior design value and advantages}

\subsection{People live in harmony with nature}

The harmonious coexistence of man and nature is the core viewpoint of ecological theory and the basic criterion of ecological interior design. Ecological interior design can reduce the loss of natural resources, reduce energy consumption, and control the timber and land occupancy rate. At the same time, every detail in the ecological interior design can reflect the concept of hello, hello, and harmony between humans and nature. Through scientific and reasonable design, humans' damage to the natural ecological balance is reduced, and this is precisely the reason. The greatest application value. For example, in traditional interior design, in order to ensure the cleanliness of the indoor environment, range hoods and other scientific and technological means are used to directly discharge polluting gases outdoors, which invisibly aggravates environmental pollution. In ecological interior design, the most effective control of this kind of pollution will be carried out through the application of green materials. At the same time, natural ventilation channels are scientifically designed to reduce indoor suffocation with better ventilation and convection, and reduce people's pollution to the outdoor environment.

\subsection{Comfortable and reasonable living conditions}

Both humans and nature are the subjects of ecological theory, and ecological interior design should not only consider the protection of the environment, but also fully consider the comfort of human living conditions. Therefore, the design respects the human's requirements for the quality of life. The requirements of humans for the living environment are not only indoor environments but also outdoor environments. A comfortable and harmonious outdoor environment can improve human happiness, and a quiet and comfortable indoor environment can enhance human sense of belonging, which is also the main pursuit of ecological interior design. . Research has found that in recent years, people have realized that conquering and defeating nature will not bring them a more comfortable living environment, but will bear huge consequences, because governance is far more difficult than destruction. Therefore, from the perspective of ecological theory, interior design should fully embody the concept of conforming to and enjoying nature, and creating a sustainable and comfortable living environment for people.

\subsection{Sustainable development of design concept}

For a long time, interior design has always been people-centered, but the traditional interior design concept puts more emphasis on meeting people's needs and desires, while the ecological interior design concept puts more emphasis 
on people-oriented and sustainable development. In the ecological interior design, through the selection of renewable materials and environmentally friendly materials, and the integration of green ecological technology, it not only meets the needs of human enjoyment, but also protects natural resources and truly achieves sustainable development.

\section{Concluding remarks}

With the continuous popularization of the concept of green ecological construction, the interior design industry should pay more attention to ecological interior design, fully realize the importance of incorporating ecological theory into interior design, and adopt modern ecological design measures based on ecological principles., To improve the environmental protection of interior design, so that people and nature can live in harmony, so as to achieve sustainable development.

\section{References}

1. Jiang Xia. Discussion on interior design from the perspective of ecological theory[J]. China Building Decoration, 2019(09): 122-123.

2. Zhang Lihua, Liu Jianhua. Research on interior decoration design based on ecological theory[J]. Ju She, 2018(34): 20.

3. Gan Lu. Research on interior design based on ecological theory[J]. China Nationalities Expo, 2017(03):140-141. 